\title{
Management of multidrug-resistant tuberculosis and patients in retreatment
}

\author{
J.A. Caminero
}

ABSTRACT: Retreatment of tuberculosis involves the management of entities as diverse as relapse, failure, treatment after default, and poor patient adherence to the previous treatment. The emergence of conditions for selection of resistance (failure and partial abandonment) is a matter of great concern.

The development of a retreatment regimen for tuberculosis requires consideration of certain basic premises. The importance of a comprehensive and directed history of drugs taken in the past, and the limited reliability of susceptibility tests to many of these drugs, should be kept in mind. Taking this into account, and possessing a thorough knowledge of all anti-tuberculosis medications, it is possible to cure almost all patients with an appropriate retreatment regimen including a minimum of three or four drugs not previously used. Nonetheless, the treatment of these patients is so complex that it should only be carried out by experienced staff.

Concern about treating tuberculosis patients with drug resistance varies greatly depending on the available resources. High-income countries should provide individual treatment regimens adapted to each patient; however, in other settings, restricted resources could justify the implementation of standardised therapeutic guidelines with second-line drugs in order to facilitate management and reduce costs.

KEYWORDS: Multidrug-resistant tuberculosis, standardised regimen, treatment, tuberculosis management

$\mathbf{P}$ overty has been the historical ally of tuberculosis (TB), and, consequently, from an economical perspective, private companies have never considered TB research as a cost-effective action. This reasoning could apply to the study of all fields of the disease, but perhaps the most affected has been that of development of new drugs with activity against Mycobacterium tuberculosis. As a result, one of the most important limitations of $\mathrm{TB}$ treatment nowadays is the limited availability of drugs. Indeed, only two drugs, isoniazid $(\mathrm{H})$ and rifampicin (R), are highly effective against this microorganism, which renders the cure of $\mathrm{TB}$ patients with resistance to $H$ and $R$ very difficult. These cases are given the specific term of multidrug-resistant (MDR) TB (MDR-TB). Management of patients with MDR-TB is extremely complicated, and studies and publications supporting one or other of the various therapeutic strategies are very rare, making these strategies the subject of recurrent controversy.

The complexity of management of patients with MDR-TB should be an indication for the treatment to be carried out exclusively by very experienced medical staff [1]. This is even more important when it is considered that, in many cases, retreatment represents the patient's last chance of a cure. However, a two-sided global problem arises here. In the first place, many professionals with restricted knowledge, facing

Previous articles in this series: No. 1: Cardona P-J, Ruiz-Manzano J. On the nature of Mycobacterium tuberculosis-latent bacilli. Eur Respir $J$ 2004; 24: 1044-1051. No. 2: Rieder H. Annual risk of infection with Mycobacterium tuberculosis. Eur Respir J 2005; 25: 181-185. No. 3: Mitchison DA. Drug resistance in tuberculosis. Eur Respir J 2005; 25: 376-379. No. 4: Kim SJ. Drug-susceptibility testing in tuberculosis: methods and reliability of results. Eur Respir J 2005; 25: 564-569. No. 5: Dlodlo RA, Fujiwara PI, Enarson DA. Should tuberculosis treatment and control be addressed differently in HIV-infected and -uninfected individuals? Eur Respir J 2005; 25: 751-757.

\section{AFFILIATIONS}

Pulmonary Medicine Dept, Hospital de Gran Canaria "Dr. Negrín", Las Palmas de G.C., Spain.

\section{CORRESPONDENCE}

J.A. Caminero

Pulmonary Medicine Dept Hospital de Gran Canaria "Dr. Negrín"

Barranco de la Ballena s/n 35020 Las Palmas de G.C. Spain

Fax: 34928450085

E-mail:

jcamlun@gobiernodecanarias.org

Received:

September 022004

Accepted:

September 282004 
an attractive subject, dare to treat these patients, worsening the problem day by day [1]. The second aspect stems from the current situation in poor countries with a very high TB burden, where patients with suspicion of MDR-TB are so numerous [2] that they cannot be managed by experienced specialists alone.

This article discusses the most controversial issues in the complex management of these patients and proposes adapted ways of treating them according to the specific epidemiological and clinical settings and available resources.

\section{WHEN TO SUSPECT MDR-TB AND TREATMENT ORIENTATION}

Two clear-cut possibilities can be encountered in the management of TB cases suspected of drug resistance: 1) patients who have never received anti-TB drugs and who have contracted drug-resistant strains of M. tuberculosis; and 2) patients who have already received one or more previous treatment regimens (i.e. patients undergoing retreatment). Retreatment patients correspond to the greater part of the problem and the major source of complications.

The management of new patients suspected of having contracted drug-resistant strains of $M$. tuberculosis should be essentially similar to the treatment of other new patients. They should receive the same initial standardised treatment recommended by the National Tuberculosis Programme (NTP); however, as resistance is highly suspected, drug susceptibility testing (DST) to first-line drugs should be carried out. If these tests confirm MDR, but the patient shows a favourable clinical progression, the initial regimen should perhaps remain unchanged, since some reports have demonstrated that 20$50 \%$ of these new patients with MDR-TB are cured by standard regimens [3]. If the clinical response is not favourable, the initial treatment must be interrupted in favour of a new regimen following the basic premises detailed later. Nonetheless, some authors advocate the administration of empirical treatments with up to seven drugs until DST results are available, with further adaptation of the regimen subsequent to these results [4].

However, the largest and most complex part of the problem encompasses patients undergoing retreatment. As the term implies, TB retreatment refers to the instauration of a treatment regimen in a patient who has previously been treated with anti-TB drugs. These patients have already had the opportunity to receive a curative treatment for the disease; therefore, excluding relapse cases, most retreatment cases arise due to errors during the implementation or administration of the previous treatment regimen [1]. In order to maximally reduce the number of patients potentially requiring retreatment, two basic conditions must be met including: following of standardised initial treatment regimens, and strict monitoring of drug intake [1].

The retreatment of TB involves microbiological and operational entities as diverse as relapse, failure, treatment after default, and poor patient adherence to the previous treatment. All of these conditions imply different approaches and must be accurately recognised by the physician who handles these problems.

\section{RETREATMENT CASES}

Bacteriological relapse is defined as the reappearance of bacterial activity in a patient who has followed and completed a correct treatment and, therefore, been cured of TB $[1,5]$. However, there can be certainty that a patient has completed all their medication only when treatment administration is directly observed. If the treatment was not supervised, the authenticity of a relapse can be questioned. The relapse is usually due to a quiescent or persistent bacterial population [6], which did not proliferate during administration of the initial treatment, and, as a consequence, the conditions necessary for selecting the spontaneously resistant mutant bacilli present in every bacillary population were not attained. For this reason, it is theoretically accepted that relapses exhibit the same pattern of resistance as that of the patient's initial TB; hence the same initial treatment regimen could be administered [1]. If the relapse occurs several years after the initial treatment, re-infection is more likely than relapse $[7,8]$. This is not simple to demonstrate, but the indication for the same initial treatment scheme still applies. Although relapses are usually due to susceptible bacilli, it is advisable to carry out DST to first-line anti-TB drugs.

However, this theory about relapses and their presumed equivalent pattern of drug susceptibility to that of the initial process applies only when the patient has correctly completed an adequate treatment regimen [1]. Indeed, when DST is conducted in the field, the incidence of resistance among relapse patients increases significantly compared to that seen in initial cases [9]. This might be explained by irregularities in drug intake but also by selection of initial single or multiple resistances. This situation has led to the recommendation that, in low- and middle-income countries and under NTP conditions, relapses be treated with a reinforced first-line drug regimen, administered for a longer period of time $[5,10]$.

Treatment failure is defined as the reappearance, whilst the patient is receiving treatment and after the negative conversion of cultures, of bacteriological activity [1, 5]. It is also considered treatment failure when cultures do not become negative during the course of treatment. For the initial 6-month treatment scheme with $\mathrm{R}$ in both phases, failure is considered when sputum cultures are still positive at the end of the fourth month. For the 8-month treatment scheme, without $\mathrm{R}$ in the second phase, failure is not diagnosed before the end of the fifth month $[1,5,10]$. The failure is due to a population of bacilli that is metabolically active [6] and proliferates during the treatment, which creates favourable conditions for selection of naturally resistant mutant bacilli present in the original population. Therefore, the failure situation should imply resistance to all of the drugs being administered at the time when failure was diagnosed [1]; hence the importance of carrying out DST to first-line drugs.

Once again, this is the theoretical explanation for failures, but, in the field, some studies show that up to $50 \%$ of failures exhibit drug susceptibility in antibiograms [11], and can be cured with an initial or reinforced first-line-drug-based scheme [3]. Here, what is known as operational failure comes into play, 
i.e. when irregularities in drug administration lead to the conclusion of treatment failure because of the absence of negative conversion of cultures, due to faulty adherence to treatment. Other contributing factors are the imperfect correlation between DST results and the behaviour of bacilli in vivo, and the extremely small fraction of patients in whom negative conversion of cultures take $>4$ months despite welldocumented susceptibility to drugs.

The third large group of patients who undergo retreatment includes those who previously abandoned their treatment. There are two different kinds of default: patients who abandon all of their drugs, and those who omit one of them. Those who abandon all of their drugs usually do not incur an elevated risk of selection of resistance, since the drugs were effective when the patient took the treatment, and there were no favourable conditions for selection of the naturally resistant mutants. When the effect of the treatment was interrupted due to default, the entire bacillary population resumes growth, as before the initial treatment. Thus, in these cases, it is adequate to reintroduce the initial treatment regimen. The situation is entirely different when the patient abandons one of the drugs while undergoing treatment. It generates the conditions for the selection of microorganisms spontaneously resistant to the drugs the patient continues taking [1].

The last group consist of patients who adhere poorly to treatment. They start treatment with a fairly regular drug intake but later fail to comply and show frequent nonattendances. This behaviour is by far more common than might be thought, particularly during the latter months of treatment, when patients must continue taking medication even though they are relieved of symptoms [1]. When this situation (days with and days without medication) continues for weeks or months, suitable conditions arise for naturally resistant mutant bacilli to develop. In this context, it is important to remember the differences between antibiotics regarding the persistence of their effects; for instance, the post-antibiotic effect of $\mathrm{H}$ is clearly longer than that of $\mathrm{R}$ [12]. In consequence, if a patient takes $\mathrm{H}$ and $\mathrm{R}$ once per week, the effect of $\mathrm{H}$ remains during the latter days of the week, whereas the effect of $\mathrm{R}$ is removed, favouring conditions for the selection of H-resistant mutants for a short period of time. When this condition recurs over a period of weeks or months, there could be selection, initially of spontaneously H-resistant mutants and later on of naturally R-resistant mutant bacilli.

However, the major therapeutic problem in TB is presented by chronic patients who have received multiple treatments and are thought to carry MDR and resistances to numerous other medications. In most cases, the blame can be placed on either the physician or the NTP, for prescribing inadequate treatment regimens. Even though the patient may be responsible for partial abandonment of the medication or poor compliance, the physician and the NTP may also be responsible for prescribing individual drugs rather than combined formulations or for failing to strictly supervise the treatment [1]. When all of the drugs are administered combined in the same tablet and the patient comes to abandon the medication, all of the drugs will be interrupted simultaneously and the selection of resistance prevented.

\section{IDENTIFICATION OF RESISTANCE IN RETREATMENT PATIENTS}

The main approaches to the identification of resistance in retreatment patients are establishing a history of previous TB treatment and DST.

The first requirement for starting a retreatment regimen is evaluation of the possibility that the patient carries resistant bacilli. From this viewpoint, it is essential to take their medical history before any other action. This directs diagnosis towards relapse, total or partial default on treatment, failure, or sustained poor compliance of the patient to the regimen. In the case of relapse or total treatment default, it can be assumed that there is no resistance and the same initial regimen could be reintroduced, with close monitoring of the bacteriological response and DST to first-line drugs [1].

However, in the case of failure, partial default on medication, sustained poor compliance of the patient, or chronicity with multiple previous treatments, the presence of resistance to one or more drugs is very likely. In these cases, identification of the patient's pattern of resistance requires two approaches: a comprehensive history of previous $\mathrm{TB}$ treatments, and the execution of DST [1, 13-15].

In order to establish a complete chronology of the previous introduction and cessation of drugs, a meticulous history of previous anti-TB drugs, including administered doses and pharmacological associations of prior regimens, should be taken by experienced staff who allow a reasonable length of time for this task ( $\geqslant 30-60 \mathrm{~min}$ ). This reconstructs the precise chronology of drug introduction and withdrawal, allowing the evaluation of real or masked monotherapies previously received by the patient. Figure 1 presents a model document for recording the patient's treatment history [1], which must be filled in for each year of treatment received by the patient. Marking the boxes corresponding to the periods during which the patient has taken each drug permits clear evaluation of the efficacy of prescribed drug combinations. Once completed, this chronology conveys very valuable information about drugs with probable resistance and others with potential current efficacy. Using this method, resistances to particular drugs can be predicted with high probability, and their inclusion in the retreatment plan avoided [1]. Surprisingly, if this treatment history is taken meticulously, not only the errors leading to

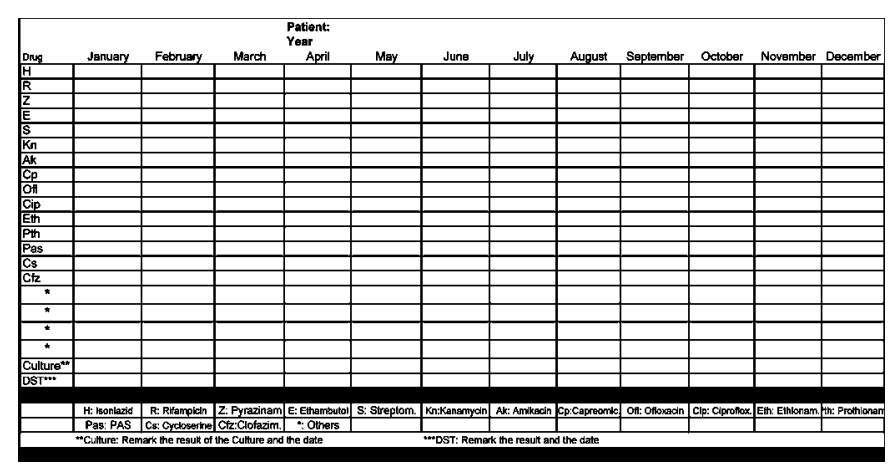

FIGURE 1. Model form for the history of drugs taken by patients with suspected or confirmed resistance to antituberculosis drugs. DST: drug susceptibility testing; PAS: $p$-aminosalicylate. 
many of the failures but also drugs with potential efficacy, despite their prior use, since they were prescribed in sound associations and led to culture conversion in the past, can be identified. In conclusion, there is no reason to expect resistance to all previously administered drugs, as it is often erroneously presumed when these patients are taken care of by inexperienced staff, or when their drug history has been taken hastily.

In practice, the quality of information collected through this medical history is better than that from DST, which can take $>3$ months (when carried out by conventional methods on solid media) and may also fail due to insufficient growth of cultures. In addition, it is important to realise that, although the in vitro and in vivo correlation of the antibiogram is very reliable for $\mathrm{H}$ and $\mathrm{R}$, its consistency is, by far, lower for other drugs [1, 15-19]. It should be pointed out that drug resistance, as detected in the antibiogram, reflects the inefficacy of this drug in culture media, but drug susceptibility does not necessarily correspond to the efficacy of the drug within a new regimen; the latter can be estimated in view of the history of previous treatments.

DST to first-line drugs should be performed systematically in all of these patients despite its drawbacks, the first of which is variable reliability: adequate for $\mathrm{H}$ and $\mathrm{R}$, but much less for streptomycin (S) and ethambutol (E) [18, 19]. DST to pyrazinamide $(Z)$ requires BACTEC, a radiometric method not available in most low-income countries. Another inconvenience is the delay in obtaining results, as retreatment cannot be delayed, when the adequate drugs are available. Conversely, DST to second-line drugs should not be systematically carried out given its difficulty, cost and poor reliability [18]. Even in wealthier countries, with multiple methods for performing DST to second-line drugs, interpretation of the results requires cautious analyses by experienced experts. Studies aimed at standardisation of the results of DST to second-line drugs are scarce and yield unequivalent results; the concentrations of each drug and definition of resistance used vary greatly even between the laboratories performing the most tests [18]. In contrast, many specialised centres routinely design or modify retreatment regimens based on DST results. Such practices should be discouraged until DST protocols are standardised and their predictive values calculated. At present, it appears that DST results concerning second-line drugs such as kanamycin (Kn) and ofloxacin (Ofl)/ ciprofloxacin could be of great help, but not DST to other drugs $[17,18]$.

In countries of low or medium income, the history of previously administered drugs as a method for resistance screening is yet more important, since DST is performed following the proportional method on solid culture media, leading to a delay of $4-5$ months in receiving the information [1].

\section{BASIS OF TB RETREATMENT REGIMEN DEVELOPMENT}

Once the comprehensive history of drugs taken in the past is completed, a treatment regimen should be designed following the 10 guidelines listed in table 1 [1]. Perhaps the most controversial item since the late 1990s has been the number of drugs required to design a good retreatment scheme with second-line drugs [1, 13, 14, 17, 20-49]. The recent recommendation published by the American Thoracic Society (ATS), Centers for Disease Control and Prevention, and Infectious Diseases Society of America has revived the debate [20]. These leading scientific societies recommend the use of four to six second-line drugs in patients carrying MDR and resistances to other first-line drugs. The rationale for this recommendation is the presumed better result obtained with this high number of drugs in three studies [21-23]. However, a careful review of the three articles shows that there was no comparison, nor demonstration of better outcomes attributable to a higher number of administered drugs [24]. The only reasonable conclusion from these articles [21-23], as from a number of others [43-49], is that acceptable results are obtained prescribing more than four drugs. Nevertheless, it does not demonstrate that the use of fewer drugs would entail inferior results. It is worth recalling that, in the period 1950-1960, when R was still not available, multiple articles reported very good results with a combination of only three drugs in patients with resistance to $\mathrm{H}$ and $\mathrm{S}$ and also to other medications [25-31, 3342]. Moreover, in many of these studies, prior to the discovery of $E$, this drug was not prescribed, making the management of these patients as complicated as that of MDR cases nowadays. This undocumented modification of ATS guidelines regarding MDR-TB patients is noteworthy, as official ATS documents dating 1965-1966 [13, 14] recommend only two or three new drugs for the management of MDR. The official document

\title{
TABLE 1 Guidelines for developing a retreatment regimen in tuberculosis
}

\author{
Retreatment should always be started in a reference centre by experts in the management of second-line drugs \\ It is essential to design a treatment based on a comprehensive patient history of prescription drugs taken in the past \\ Confirm MDR with a susceptibility test to $\mathrm{H}+\mathrm{R}$; evaluate results of susceptibility test to $\mathrm{Kn}$ and ofloxacin/ciprofloxacin \\ Combine a minimum of three or four drugs never used before or probably devoid of resistance (adequate associations in previous studies) \\ Prescribe a maximum of bactericidal drugs having effects on different targets of the bacillus \\ Always include an aminoglycoside or capreomycin \\ Beware of possible cross-resistance between drugs, particularly: aminoglycosides ${ }^{\#}$; all the quinolones; and low-level resistance to $\mathrm{H}$ and ethionamide \\ Minimum length of treatment: 18 months without $\mathrm{H}$ and $\mathrm{R}$; and 12 months if $\mathrm{H}$ or $\mathrm{R}$ can be used \\ Close supervision of treatment \\ Never add only one drug to a regimen having demonstrated previous inefficiency
}

MDR: multidrug resistance; $\mathrm{H}$ : isoniazid; R: rifampicin; Kn: kanamycin. ${ }^{*}$ : resistance to the aminoglycoside group is considered to be unidirectional; therefore, they should be prescribed sequentially in the order: streptomycin, kanamycin, and finally amikacin. See Basis of TB retreatment regimen development section for explanation. 
from 1994 has stated a slight change in guidelines, recommending the use of at least three new drugs, although no references justified this modification [32]. Prescription of more than four drugs is unjustified from a bacteriological standpoint and favours intolerance, which may lead the patient to spontaneous or medically advised abandonment of treatment, owing to severe side-effects [21-24].

In consequence, a good retreatment regimen with second-line drugs requires no more than three or four drugs that the patient has certainly never received before [24]. This regimen should be continued for 18-24 months with at least two or three of these drugs in cases where $\mathrm{H}$ or $\mathrm{R}$ is not useful (if they can be used, 12 months of treatment should suffice). Such treatment should be prescribed in specialised centres by experienced staff, since most second-line drugs are difficult to handle and present a higher frequency of side-effects $[1,4$, $15,20]$.

The following points clarify certain aspects regarding the drugs to be included in a retreatment regimen and the guidelines presented in table 1 .

1. It is important to assign the greatest possible number of available bactericidal drugs so as to have effects on different targets of the microorganism. If few are available, there should always be an aminoglycoside. Capreomycin does not belong to this group but shares a similar mechanism of action and therefore could be considered as a choice along with the aminoglycosides. In addition, capreomycin, being less toxic and well tolerated, is recommended when $S$ cannot be used.

2. It is important to consider the possibility of cross-resistance to improperly prescribed drugs. Resistance to the aminoglycoside group is considered unidirectional; therefore, they should be prescribed sequentially in the order: $S, K n$, and amikacin (table 1). If there is resistance to $S$, sensitivity to the others is probably conserved; when resistance to $\mathrm{Kn}$ is present, susceptibility to the others, except for S, is very likely. There is also cross-resistance between $\mathrm{R}$ and the ansamycins and between all of the quinolones.

3. When resistance to one drug is documented or suspected, this drug should be excluded from the new regimen. The drug might be considered for the treatment regimen only when no other medication is available.

Antimicrobial agents with demonstrated activity against $M$. tuberculosis, which are suitable for a retreatment regimen, are listed in table 2 [1]; the great majority of second-line drugs are expensive and difficult to obtain. As shown in table 2, there are $\geqslant 13$ drugs with demonstrated anti-mycobacterial activity, which have already been used in various studies. However, the efficacy of many of them is inadequate, and their bactericidal and sterilising abilities differ. It is important to use as many first-line drugs (H, R, E, S and Z) as possible, since, beside being more efficacious, they show better tolerance and are more readily available on the market [1].

As has been discussed, whenever the available choice of drugs permits, the great majority of TB patients can be cured using three or four drugs never received previously. In the end, the most important restrictions are defined by the availability of and capability of obtaining some of these drugs, experience in

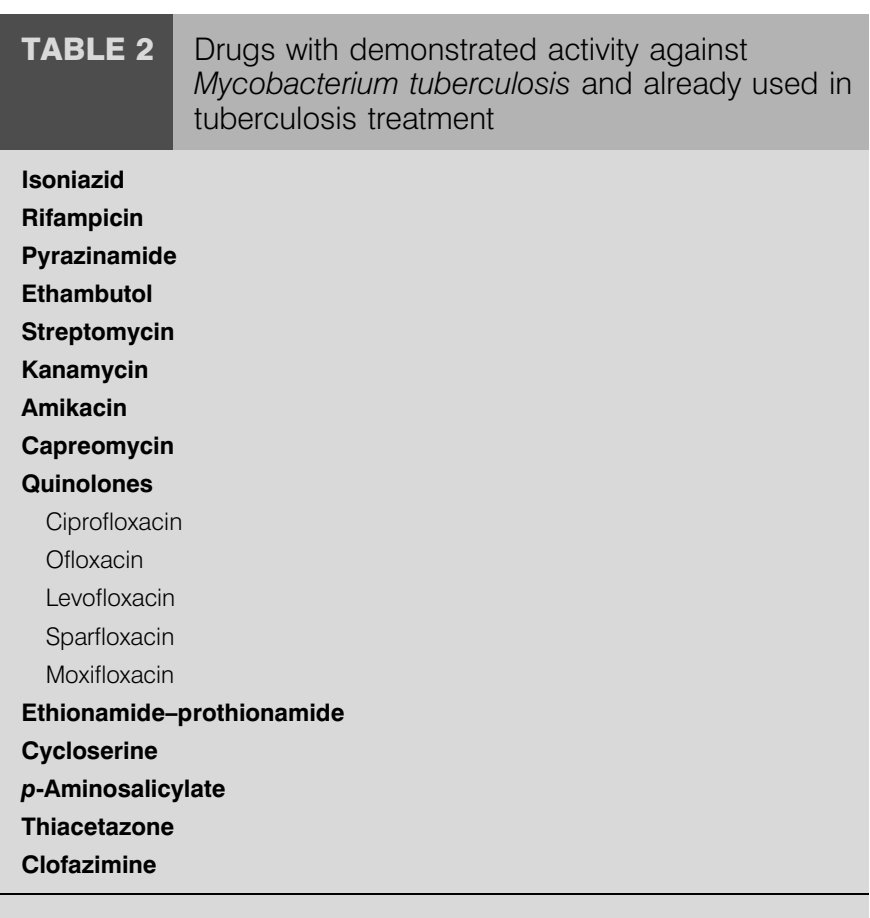

the design of appropriate treatment regimens, and the management of these more toxic and poorly tolerated drugs [1]. After all that has been said, it is obvious that the treatment of MDR-TB should practically never resort to surgery. Moreover, surgery requires localised lesions, good functional respiratory capacity and the absence of available drugs for designing a treatment regimen in keeping with the above recommendations. It should be emphasised that the surgical solution involves high morbidity and mortality and does not always cure the disease. All things considered, retreatment of TB becomes a question of appropriate management of secondline drugs [1].

\section{FINANCIAL RESOURCES AND TB RETREATMENT}

This important section should start by emphasising the extreme importance of the prevention of resistance dissemination. The best approach to preventing the delicate and difficult problem of MDR is the development of adequate initial regimens and close supervision of treatments. Up to this point, this article has covered the state of the art as regards this subject, readily applicable to the best economic, sanitary and epidemiological settings as encountered in the highest income countries. These nations bear a very low burden of TB and benefit from plentiful economic and sanitary resources. They have at their disposal more specialised physicians than patients admitted for TB retreatment, and, since there is no financial restriction, they provide each patient with individualised and expert care, multiple diagnostic tools (rapid DST) and all the existing therapeutic choices. On the one hand, these medical experts identify relapses and total defaults on treatment and start the same initial treatment regimen, until the results of the DST are available; and, on the other hand, they identify failures and partial defaults on treatment and design retreatment regimens in which they include suitable second-line drugs. On these grounds, it is recommended, in 
industrialised countries, that a proper individualised retreatment scheme be applied for each case $[1,15,20]$. It is noteworthy, however, that these regimens cost in the range US $\$ 5,000-10,000$, and can reach US $\$ 100,000$ when they include extended hospital stays.

These conditions do not prevail in most of the world [2]. In countries with a high TB burden and limited economic resources, even when an efficient NTP has been implemented, it is estimated that $10-15 \%$ of initial patients, relapse, failure and default cases, will sooner or later return to the programme [50-53]. This means that, if a country diagnoses 10,000 TB cases $\cdot \mathrm{yr}^{-1}, 1,000-1,500$ cases $\cdot \mathrm{yr}^{-1}$ would require retreatment. Depending on the country's economic and social standards, the number of patients can be 50-500 times greater than the number of specialised physicians available. Clearly, it would be impossible for each patient admitted to the programme to be taken care of by an expert in the field. For an inexperienced care provider, the risk of error in the process of differentiating the four types of case that require retreatment (relapse, failure, treatment after default, or patients with previous poor compliance) is high [1]. It is essential to discriminate between these four situations, since only failure entails a high probability of resistance, even if poorly compliant patients could also carry some. The rest of the cases, amounting to $>90 \%$ of patients requiring retreatment, correspond to relapses or total defaults on treatment and are assumed to be at low risk of having selected resistant bacilli, as previously explained [1].

Considering all of the above, and given the substantial number of cases admitted for retreatment in countries with a high TB burden, such patients have to be treated at a peripheral level in the health system, where conditions are not optimal for discriminating between the possible conditions leading to retreatment. Furthermore, it would be impossible to meet the enormous expenses related to individualised retreatment for such a large number of patients. It is worth comparing the cost of retreatment of US\$5,000-10,000 to the cost of the initial treatment of $\sim$ US $\$ 10$. This means that the expenses for one individualised retreatment could be enough to fund the initial treatment of 500-1,000 patients, which represents an authentic epidemiological priority. Moreover, most countries in which TB is highly endemic can devote to healthcare no more than US\$50-100 per capita.

Operational (inability to discriminate between the conditions resulting in retreatment at the peripheral level of healthcare) and economic restrictions led the International Union Against Tuberculosis and Lung Disease and the World Health Organization to conclude that the best solution consists of implementing a standard retreatment scheme with first-line drugs, systematically applicable to all patients admitted to the programme with a previous history of TB treatment [5, 10]. This 8-month regimen, administered in three phases (HRZES for 2 months (2HRZES) $/ 1 \mathrm{HRZE} / 5 \mathrm{H}_{3} \mathrm{R}_{3} \mathrm{E}_{3}$ (subscript indicates number of weekly doses)), and which must be strictly supervised, only costs US\$40-60. This is an operational scheme to be applied under similar management conditions as those for initial patients, i.e. at the peripheral level and not necessarily by expert staff. In the field, this regimen can cure most relapses and treatment defaults (the most common sources of patients for retreatment) and a variable proportion of initial failures. The downside of this regimen, as discussed below, is the treatment of failures, which fortunately represent the smallest group. In this way, in countries with a low rate of initial MDR and an efficient NTP, the majority of patients requiring retreatment (defined exclusively by the fact that they have been previously treated) will be cured at the peripheral level. It will also be possible to identify those patients who carry resistances (i.e. who are not cured), accounting for $<1-$ $2 \%$ of all initial patients. This percentage may be higher where the above conditions are not fulfilled. This reduced number of patients could then be treated and given a greater level of attention by specialised physicians, able to prescribe secondline drugs [1].

As mentioned above, the difficulty with the 8-month regimen is the group representing failure of the initial scheme. Typically, a high percentage of these failures may have induced selection of resistance to the initial treatment drugs, although several clinical trials have shown that there was susceptibility to $>50 \%$ of the drugs [11]. Therefore, in the case of failure of an initial 6-month regimen (2HRZE/4HR), there is a high risk of resistance to $\mathrm{H}$ and $\mathrm{R}$ and so, if an 8-month regimen (2HRZES/1HRZE $/ 5 \mathrm{H}_{3} \mathrm{R}_{3} \mathrm{E}_{3}$ ) is prescribed, it would amount to masked monotherapy with $\mathrm{E}$ during the last 5 months of treatment. This entails a high risk of amplifying resistance to $\mathrm{E}$ and will not cure the patient. Likewise, if the patient has been initially infected with an MDR strain of $M$. tuberculosis (primary MDR) and fails to respond to the initial 6month regimen, selection of resistance to $E+Z$ during the initial 2 months of treatment is very likely, because the combination of these two drugs is weak, in the context of inefficiency of $\mathrm{H}$ and $\mathrm{R}$. In this case, administration of the 8-month treatment regimen would be equivalent to masked monotherapy with $S$ in the initial phase, with a high risk of amplifying resistance to this antibiotic. The attitude of applying the 8-month retreatment regimen to patients showing failure of the initial scheme favours the amplification of resistance and should be avoided when adequate resources become available, adapting these guidelines to the situation and the country's economy. If a country is so poor that only first-line drugs are available, the 8month regimen would apply only to previously treated patients, accepting, in terms of cost-efficiency, the risk of amplification of resistance to $\mathrm{E}$ or $\mathrm{S}$ in a significant percentage of the failures as regards the initial regimen. In so doing, the country will accumulate a series of patients showing resistance to all first-line drugs, and thus, creating the need for a new solution in the future. These patients with failure of the initial regimen should benefit from a standardised scheme with second-line drugs, as soon as the country has enough resources to conduct DST to first-line drugs in all cases of treatment failure and qualifies with a minimal reserve of second-line drugs. Only countries demonstrating susceptibility to first-line drugs in most of the failures may escape this recommendation [11].

Figures 2 and 3 present an algorithm for retreatment including all the possibilities applying to low- and middle-income countries. This algorithm, concerning management of the standardised and individualised schemes with second-line drugs, can also be extrapolated to most industrialised countries. 


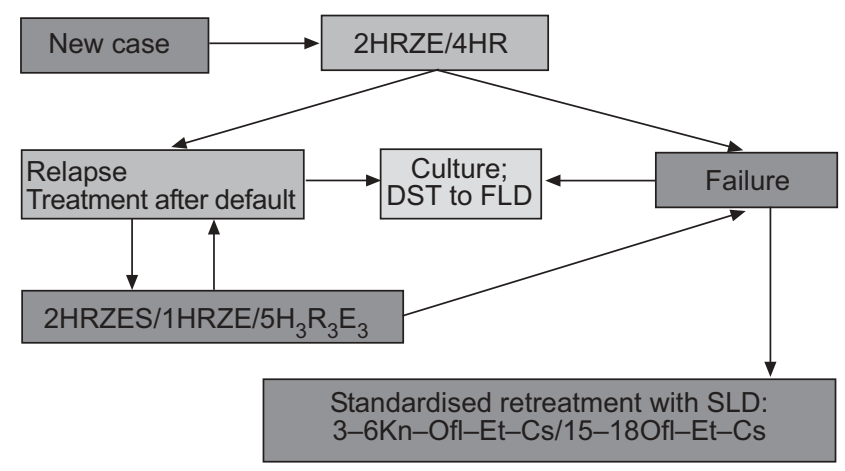

FIGURE 2. Proposal for standardised retreatment in low- and middle-income countries. H: isoniazid; R: rifampicin; Z: pyrazinamide; E: ethambutol; HRZE: HRZE for $n$ months (subscript indicates number of weekly doses); DST: drug susceptibility testing; FLD: first-line drugs; S: streptomycin; SLD: second-line drugs; Kn: kanamycin; Ofl: ofloxacin; Eth: ethionamide; Cs: cycloserine.

\section{TB RETREATMENT WITH SECOND-LINE DRUGS: STANDARDISED VERSUS INDIVIDUALISED REGIMENS}

Once patients showing resistance have been identified via the approaches outlined above, the next question is how to treat them: application of an individualised retreatment scheme according to the DST pattern of resistance $[4,15,20,54]$; or implementation of a standardised retreatment regimen with second-line drugs $[5,17,55,56]$, avoiding DST. There has been a great deal of controversy regarding these two approaches $[4,5$, $15,17,20,54-57]$, particularly since many low-income countries have rarely used second-line drugs, which implies very little resistance to them. At this point, it is important to consider that patients with MDR could stem from two different sources, entailing very different diagnostic and therapeutic procedures [17]: an efficient NTP, having received an adequate regimen with first-line drugs; and multiple treatment schemes, including second-line drugs, without adequate treatment supervision.

\section{MDR patients proceeding from an efficient NTP after adequate treatment with first-line drugs}

In such cases, where patients have only received standardised regimens with first-line drugs, under the supervision of an efficient NTP, failure should not be associated with resistance to second-line drugs. These cases could be designated incidental or prospective, and, ideally, should all be treated using the same retreatment regimen (fig. 2). Perhaps the best scheme would be 3-6Kn-Ofl-ethionanide (Eth)-cycloserine (Cs)/15-18Ofl-Eth-Cs, for the following reasons: it includes the best known and least toxic combinations (although more toxic than first-line regimens); it is the least expensive; all of these drugs are readily available; and, above all, it prevents improvisations, which tend to occur with individualised treatments $[1,17]$. In any case, this standardised scheme should be adaptable to any region in accordance with the drugs prescribed there in the past. Availability of reliable data on $\mathrm{H}$ and $R$ resistance in new patients and data on failures of the initial regimen could be of help in designing this scheme.

Incidental MDR cases require neither the use of five or six drugs, nor the extension of aminoglycoside administration for longer than 3-6 months, since efficacy would remain unchanged and treatment may result in higher toxicity and cost $[1,17]$. Unfortunately, the few studies that have evaluated this regimen [55] combined these incidental MDR cases and patients who had received multiple previous treatments in the same cohort for analysis, even though the latter required a completely different attitude, as discussed below. This represents a major methodological error and should be kept in mind, as it leads to underestimation of the results of standardised regimens. Nonetheless, the excellent results achieved using a similar scheme in a country as complex as South Africa [56] are a clear example of the potential performance of this strategy.

\section{Multiple treatment schemes without adequate supervision}

These are the most complex cases. Many of them have certainly undergone multiple therapeutic schemes, not always standardised, and incur an elevated risk of resistance to all first-line drugs and some second-line drugs [17]. Indeed, management of these patients, as for the failures of a standardised regimen with second-line drugs, as described before, should be decided on a case-by-case basis (fig. 3) [1, 17]. The selection of the scheme for each patient should be founded on the patient's drug history and results of DST to first-line drugs, and Kn and Ofl/ciprofloxacin, when they are available.

Here, faced with the likelihood of resistance to several drugs, it would be justifiable to prescribe five or six drugs during the

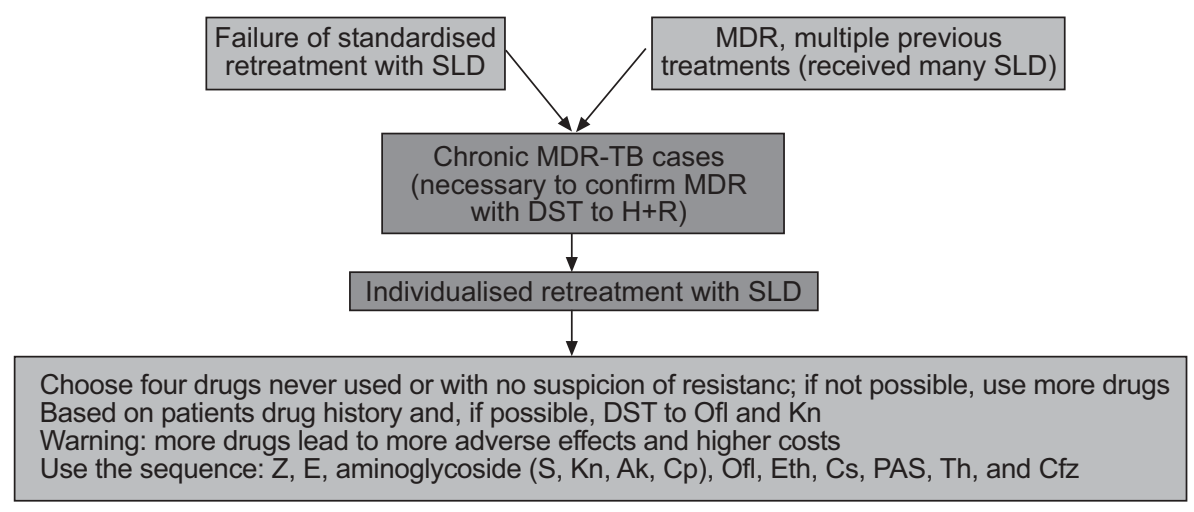

FIGURE 3. Proposal for individualised retreatment in low- and middle-income countries. SLD: second-line drugs; MDR: multidrug resistance; TB: tuberculosis; DST: drug susceptibility testing; H: isoniazid; R: rifampicin; Ofl: ofloxacin; Kn: kanamycin; Z: pyrazinamide; E: ethambutol; S: streptomycin; Kn: kanamycin; Ak: amikacin; Cp: capreomycin; Eth: ethionamide; Cs: cycloserine; PAS: p-aminosalicylate; Th: thiacetazone; Cfz: clofazimine. 
first phase of these regimens. Understandably, the use of a higher number of drugs in these patients offers a greater chance of success, but also a higher rate of side-effects and the consequent defaults on treatment [17, 21-24]. In any event, if a comprehensive drug history were a requirement for admitting these patients to a retreatment scheme, it would certainly help to reduce the number of drugs required for the regimen. When no other drugs are available for these patients, the use of aminoglycosides can be extended up to 12 or 24 months, with close monitoring of side-effects.

\section{CONCLUSION}

The present article discusses and proposes standardisation of the difficult and controversial management of patients suspected of or actually carrying confirmed multidrugresistant tuberculosis, taking into account the available resources. Since this is clearly an emerging problem in many countries, it is important to simplify its management and gradually incorporate the treatment scheme into the procedures applied in the National Tuberculosis Programmes.

\section{REFERENCES}

1 Caminero JA. Guía de la Tuberculosis para Médicos Especialistas. [Tuberculosis Guide for Specialist Physicians]. Paris, International Union Against Tuberculosis and Lung Disease, 2003.

2 Espinal MA, Laszlo A, Simonsen L, et al. Global trends in resistance to antituberculosis drugs. N Engl J Med 2001; 344: 1294-1303.

3 Espinal MA, Kim SJ, Suarez PG, et al. Standard shortcourse chemotherapy for drug-resistant tuberculosis: treatment outcomes in 6 countries. JAMA 2000; 283: 2537-2545.

4 Partners in Health, Program in Infectious Disease and Social Change, Harvard Medical School. The PIH Guide to the Medical Management of MultidrugResistant Tuberculosis. Boston, MA, Partners in Health, 2003.

5 World Health Organization. Treatment of Tuberculosis. Guidelines for National Programmes. 3rd Edn. Geneva, World Health Organization, 2003.

6 Mitchison DA. Basic mechanisms of chemotherapy. Chest 1979; 76: 771-781.

7 Caminero JA, Pena MJ, Campos-Herrero MI, et al. Exogenous reinfection with tuberculosis on a European island with a moderate incidence of disease. Am J Respir Crit Care Med 2001; 163: 717-720.

8 van Rie A, Warren R, Richardson M, et al. Exogenous reinfection as a cause of recurrent tuberculosis after curative treatment. N Engl J Med 1999; 341: 1174-1179.

9 Valenzuela P, Piffardi S, Lepe R, Velasco M. Panorama actualizado de la resistencia a fármacos antituberculosos. Chile - 1998. [Updated situation of antituberculous drug resistance. Chile - 1998]. Rev Chil Infect 1998; 15: 225-233.

10 Enarson DA, Rieder HL, Arnadottir T, Trébucq A. Management of Tuberculosis. A Guide for Low Income Countries. 5th Edn. Paris, International Union Against Tuberculosis and Lung Disease, 2000.

11 Tuberculosis Research Centre, Indian Council of Medical Research. Low rate of emergence of drug resistance in sputum positive patients treated with short course chemotherapy. Int J Tuberc Lung Dis 2001; 5: 40-45.

12 Mitchison DA. How drug resistance emerges as a result of poor compliance during short course chemotherapy for tuberculosis. Int J Tuberc Lung Dis 1998; 2: 10-15.

13 American Thoracic Society. Chemotherapy of pulmonary tuberculosis in adults: The choice of drugs in relation to drug susceptibility. Am Rev Respir Dis 1965; 92: 508-512.

14 American Thoracic Society. Treatment of drug-resistant tuberculosis. Am Rev Respir Dis 1966; 94: 125-127.

15 Iseman MD. Treatment of multidrug-resistant tuberculosis. N Engl J Med 1993; 329: 784-791.

16 Ortega A. Retratamientos en tuberculosis. [Retreatment in tuberculosis]. Rev Clin Esp 1987; 180: 269-274.

17 Caminero JA. Data not published.

18 Kim SJ. Drug-susceptibility testing in tuberculosis: methods and reliability of the results. Eur Respir J 2005; 25: 564-569.

19 Canetti G. Present aspects of bacterial resistance in tuberculosis. Am Rev Respir Dis 1965; 92: 687-703.

20 AmericanThoracic Society/Centers for Disease Control and Prevention/Infectious Diseases Society of America. Treatment of tuberculosis. Am J Respir Crit Care Med 2003; 167: 603-662.

21 Goble M, Iseman MD, Madsen LA, Waite D, Ackerson L, Horsburgh R. Treatment of 171 patients with pulmonary tuberculosis resistant to isoniazid and rifampin. $N$ Engl J Med 1993; 328: 527-532.

22 Park SK, Kim CT, Song SD. Outcome of chemotherapy in 107 patients with pulmonary tuberculosis resistant to isoniazid and rifampin. Int J Tuberc Lung Dis 1998; 2: 877-884.

23 Geerligs WA, Altena R, de Lange WCM, van Soolingen D, van der Werf TS. Multidrug-resistant tuberculosis: longterm treatment outcome in the Netherlands. Int J Tuberc Lung Dis 2000; 4: 758-764.

24 Caminero JA, March P. Statements of ATS, CDC, and IDSA on treatment of tuberculosis. Am J Respir Crit Care Med 2004; 169: 316-317.

25 British Tuberculosis Association. A Report from the Research Committee of the British Tuberculosis Association. Ethionamide, pyrazinamide and cycloserine in the treatment of drug-resistant pulmonary tuberculosis. Tubercle 1963; 44: 195-214.

26 Jancik E, Zelenka M, Tousek, Makova M. Chemotherapy for patients with cultures resistant to streptmomycin, isoniazid and PAS. Tubercle 1963; 44: 443-445.

27 de March P, Burell J. Resultados obtenidos mediante regimees de asociación con dos o tres drogas secundarias en el retratamiento del tuberculoso pulmonar crónico. [Results obtained with regimes with two or three secondline drugs in the retreatment of patients with chronic tuberculosis]. Rev Clin Esp 1968; 109: 119-126.

28 Kass I. Chemotherapy regimes used in retreament of pulmonary tuberculosis. Part II. Observations on the efficacy of combinations of kanamycin, ethionamide and either cycloserine or pyrazinamide. Tubercle 1965; 46: 151-165.

29 Kass I. Chemotherapy regimes used in retreatment of pulmonary tuberculosis. Part I. Observations on the efficacy of ethambutol, capreomycin and companion 
drugs, including 4-4 diisoamyloxy-thiosemicarbanilide. Tubercle 1965; 46: 166-77.

30 Tousek J, Jancik E, Zelenca M, Jancikova-Makova M. The results of treatment in patients with cultures resistant to streptomycin, isoniazid and PAS: a five-year follow-up. Tubercle 1967; 48: 27-32.

31 Fischer DA, Lester W, Dye WE, Moulding TS. Retreatment of patients with isoniazid-resistant tuberculosis: analysis and follow-up of 146 cases. Am Rev Respir Dis 1968; 97: 392-398.

32 American Thoracic Society. Treatment of tuberculosis and tuberculosis infection in adults and children. Am J Respir Crit Care Med 1994; 149: 1359-1374.

33 Lester W, Fischer DA, Moulding T. Chemotherapy results in 257 consecutively studied cases of drug resistant tuberculosis. Am Rev Respir Dis 1967; 96: 142.

34 Lester W, Fischer DA, Dye WE. Evaluation of capreomycin and ethambutol in retreatment of pulmonary tuberculosis. Ann N Y Acad Sci 1966; 135: 890-903.

35 Pines A. Treatment of pulmonary tuberculosis with cultures resistant to two or more drugs: a series of 44 patients. Tubercle 1965; 46: 131-142.

36 Somner AR, Brace AA. Late results of treatment of chronic resistant pulmonary tuberculosis. BMJ 1966; 1: 775-778.

37 Sommer AR, Brace AA. Ethionamide, pyrazinamide and cycloserine used successfully in the treatment of chronic pulmonary tuberculosis. Tubercle 1962; 43: 345-359.

38 East African/British Medical Research Council. Streptomycin plus PAS plus pyrazinamide in the treatment of pulmonary tuberculosis in East Africa. Tubercle 1971; 52: 191-198.

39 East African/British Medical Research Council. Streptomycin plus PAS plus pyrazinamide in the treatment of pulmonary tuberculosis in East Africa: second report. Tubercle 1973; 54: 283-290.

40 Zierski M. Treatment of patients with culture resistant to the primary antituberculous drugs. Tubercle 1964; 45: 96-100.

41 Doust JY, Moatamed F. Retreatment program in pulmonary tuberculosis with resistant mycobacteria in South of Iran. Dis Chest 1967; 52: 21-25.

42 Giunio N. Chemotherapy of previously treated patients with pulmonary tuberculosis. Tubercle 1964; 45: 202-204.

43 Telzak EE, Sepkowitz K, Alpert P, et al. Multidrug-resistant tuberculosis in patients without HIV infection. $N$ Engl J Med 1995; 333: 907-911.

44 Narita M, Alonso P, Lauzardo M, Hollender ES, Pitchennik AE, Ashkin D. Treatment experience of multidrug-resistant tuberculosis in Florida, 1994-1997. Chest 2001; 120: 343-348.
45 Tahaoglu K, Torun $\mathrm{T}$, Sevim $\mathrm{T}$, et al. The treatment of multidrug-resistant tuberculosis in Turkey. $N$ Engl J Med 2001; 345: 170-174.

46 Avendano M, Goldstein RS. Multidrug-resistant tuberculosis: long term follow-up of 40 non-HIV-infected patients. Can Respir J 2000; 7: 383-389.

47 Yew WW, Chan CK, Chau CH, et al. Outcomes of patients with multidrug-resistant pulmonary tuberculosis treated with ofloxacin/levofloxacin-containing regimes. Chest 2000; 117: 744-751.

48 Kim HJ, Hong YP, Kim SJ, Lew WJ, Lee EG. Ambulatory treatment of multidrug-resistant pulmonary tuberculosis patients at a chest clinic. Int J Tuberc Lung Dis 2001; 5: 1129-1136.

49 Mitnick C, Bayona J, Palacios E, et al. Community-based therapy for multidrug-resistant tuberculosis in Lima, Peru. N Engl J Med 2003; 348: 119-128.

50 Programa Nacional de Control de la Tuberculosis. Situación de la tuberculosis en El Salvador, 2001. [National Tuberculosis Control Programme. Tuberculosis Situation in El Salvador, 2001]. San Salvador, Ministry of Public Health, Republic of El Salvador, 2002.

51 Programa Nacional de Control de la Tuberculosis. Situación de la tuberculosis en República Dominicana, 2002. [National Tuberculosis Control Programme. Tuberculosis Situation in Dominican Republic, 2002]. Santo Domingo, Ministry of Public Health, Dominican Republic, 2003.

52 Programa Nacional de Control de la Tuberculosis. Situación y control de la tuberculosis en Bolivia, 19992000. [National Tuberculosis Control Programme. Tuberculosis Situation and Control in Bolivia, 1999-2000]. La Paz, Ministry of Public Health, Republic of Bolivia, 2001.

53 Programa Nacional de Control de la Tuberculosis. Situación de la Tuberculosis en Honduras y estrategias de control. [National Tuberculosis Control Programme. Tuberculosis Situation and Control Strategies in Honduras]. Tegucigalpa, Ministry of Public Health, Republic of Honduras, 2002.

54 (E.A. Nardell, Division of Medicine, Harvard Medical School, Cambridge Hospital, Cambridge, MA, USA; personal communication).

55 Suarez PG, Floyd K, Portocarrero J, et al. Feasibility and cost-effectiveness of standardised second-line drug treatment for chronic tuberculosis patients: a national cohort study in Peru. Lancet 2002; 359: 1980-1989.

56 (K. Weyer, National Tuberculosis Research Programme, Medical Research Council, Pretoria, South Africa; personal communication).

57 Personal communication from the Group on DOTS-Plus for MDR-TB. 34th IUATLD World Conference on Lung Health, Paris, 2003. 